\title{
Acute heart failure (suspected or confirmed): Initial diagnosis and subsequent evaluation with traditional and novel technologies*
}

\author{
Ahmad Shoaib", Thato Mabote, Mohamed Zuhair, Xenophon Kassianides, John G. F. Cleland \\ Department of Cardiology, University of Hull, Castle Hill Hospital Kingston upon Hull, UK \\ Email: "hyas11@hyms.ac.uk, \#Ahmad.Shoaib@hey.nhs.uk
}

Received 3 April 2013; revised 12 May 2013; accepted 24 May 2013

Copyright (C) 2013 Ahmad Shoaib et al. This is an open access article distributed under the Creative Commons Attribution License, which permits unrestricted use, distribution, and reproduction in any medium, provided the original work is properly cited.

\begin{abstract}
Acute or worsening heart failure is a common reason for hospitalization which carries a high mortality. Prompt diagnosis or exclusion of $\mathrm{HF}$ and its cause and precipitating factors can improve the quality and efficiency of care, shorten hospital stay, reduce readmission and improve prognosis. While the clinician remains central to reaching a clinical diagnosis of heart failure, the use of traditional and novel diagnostic technologies will improve the specificity and sensitivity of the diagnosis of heart failure diagnosis and provide insights into its pathophysiogical profile and help tailor therapy to individual patient need. Chest $\mathrm{X}$-rays and electrocardiograms are generally available; echocardiograms less so. Novel technologies include both invasive and non-invasive methods to detect increases in intrathoracic fluid, pulmonary congestion, left ventricular filling pressures, cardiac output and vascular function. However, few of these technologies have been subjected to randomised controlled trials investigating their ability to improve patient management.
\end{abstract}

Keywords: Heart Failure (HF); Acute Heart Failure Syndrome; AHFS; Bio-Impedance; Diagnosis; Monitoring

\section{INTRODUCTION}

Heart failure (HF) is common and as longevity increases its incidence and prevalence will rise [1]. Most cases of heart failure are first diagnosed during an episode of hospital care [2] and heart failure is one of the most common reasons for emergency admission, with about $20 \%$

\footnotetext{
"No known conflicts of interest are associated with this article. \#Corresponding author.
}

of cases being new-onset and $80 \%$ an acute exacerbation of chronic HF [3]. The diagnosis may often be initially unclear, requiring further investigation, the passage of time or both to make the diagnosis with some certainty. Even so, controversy surrounds the definition of heart failure. Admissions for heart failure are often prolonged and recurrent, leading to high rates of hospital bed occupancy. In the USA, where length of stay is short, hospital mortality is around $5 \%$ and the rate of readmission within 30 days is high (25\%). [4]. In Europe, where length of stay is two or three times as long, hospital mortality is between $10 \%-20 \%$ but 30 -day readmission rates are lower at around $10 \%-15 \%, 30 \%$ - 50\% of which will be due to worsening HF [3,5]. More than $50 \%$ of patients will either die or be readmitted in the year subsequent to discharge [6].

Surprisingly, the epidemiological data on the precise presentation of acute heart failure is remarkably difficult to come by. Most authorities assume that breathlessness is the main presenting symptom and that most patients are acutely distressed by it at rest. Recent data from a National Audit of England \& Wales has called this assumption into question. Many patients present with worsening exertional breathlessness and peripheral oedema but are comfortable at rest [3,7] Also, recent reports suggest that many patients have worsening symptoms and evidence of congestion for several days prior to presentation suggesting that technologies, such as home telemonitoring, might be deployed for early detection and that redesign of services might prevent a large proportion of admissions [8-10].

There is often also a great initial uncertainty about whether HF is the cause of symptoms. Many patients are treated with diuretics without the clinician making a conscious diagnosis of HF, leading to deficiencies in investigation and treatment. Unfortunately, such patients, who may be far more common than those labelled as CHF, 
will not be picked up by most audits of heart failure [11] Therefore, most cases of heart failure may be epidemicologically "silent"; they are just treated with diuretics but never given the diagnostic "label" of heart failure [12].

Early assessment and prompt diagnosis or exclusion of HF will improve the quality and efficiency of care, shorten hospital stay, reduce readmission and improve prognosis [13]. Accordingly, there is great interest in finding new tools for early and precise diagnosis [6].

\section{CLINICAL EVALUATION OF ACUTE HEART FAILURE (AHF)}

\subsection{Purpose}

Patients with breathlessness or oedema or those treated with loop diuretics for uncertain cause should be assessed to identify

a) Whether they have heart failure and whether it is the cause of their symptoms and signs;

b) Other medical conditions (e.g.: atrial fibrillation, acute coronary syndrome, infection, anaemia or chronic kidney disease) that should be considered as a differential diagnosis, exacerbating factors or co-morbidity;

c) Their dominant acute problem (breathlessness or peripheral oedema);

d) Their cardiac phenotype (e.g.: left ventricular ejection fraction, valve disease) that will determine what treatment they should receive;

e) Their heart rate and rhythm and blood pressure that will also guide treatment.
Patients with the acute onset of severe dyspnoea often present in the early morning hours. In many healthcare systems, this is when the most junior and least experienced staff will be available. Although experts may be able to manage patients without the reassurance of technical support, less experienced staff may welcome investigations that provide them with the confidence they might otherwise lack. However, experienced staff also need to critically review their practice. The outcome of acute heart failure both in hospital and after discharge is often poor and this may be due, in part, to the persistence of outmoded concepts and practice in the care of these patients.

\subsection{Clinical Features}

Exacerbations of heart failure cover a wide spectrum of presentations with two distinctly different clinical phenotypes as shown in Figure 1. Patients may present with increasing peripheral oedema due predominantly to right or bi-ventricular failure; these patients may be termed "bloaters". They are often Comfortable at Rest but Breathless On Slight Exertion (CARBOSE). Others present with Severe Shortness of Breath at Rest (SSOBAR) which is due to a high left atrial pressure and pulmonary congestion; these patients might be termed "puffers". SSOBAR usually reflects left ventricular failure due to rapid atrial fibrillation, a high systemic vascular resistance or acute ventricular damage due to ischaemia or infarction but can be due to mitral regurgitation or other

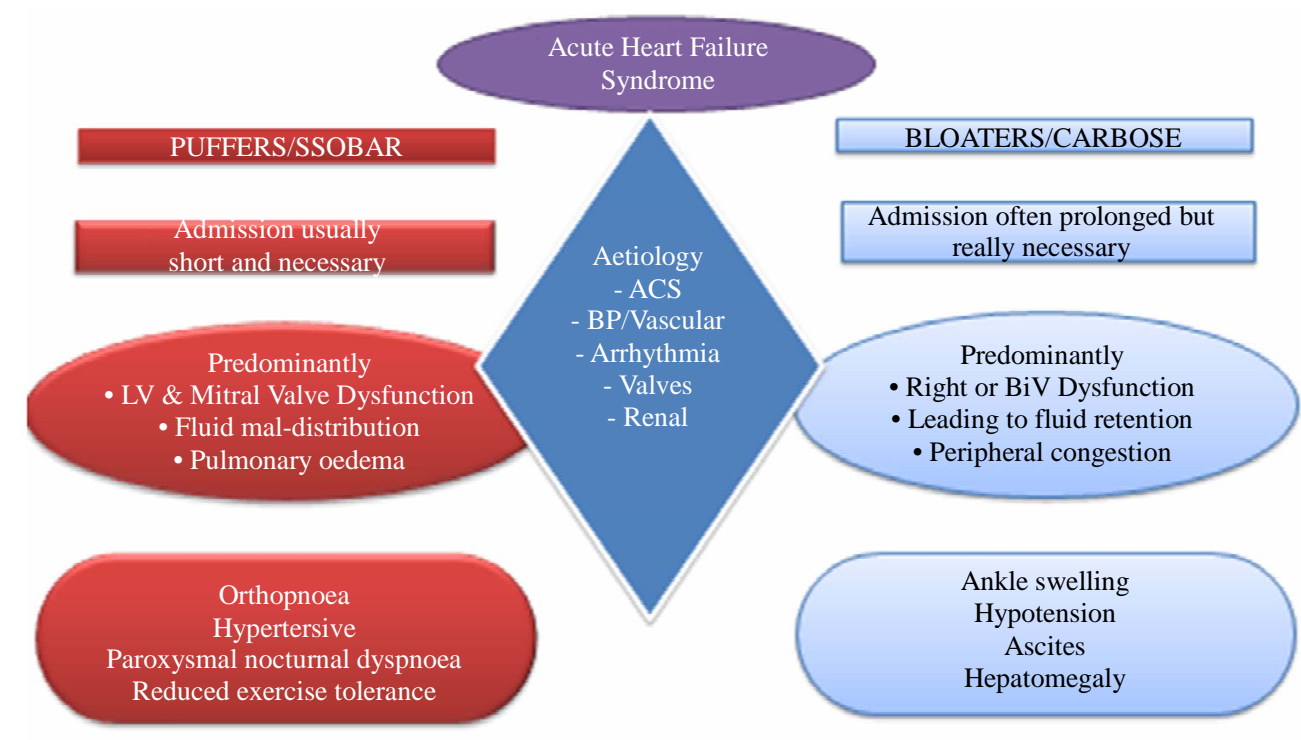

See legend for explanation of abbreviations

Figure 1. AHFS phenotypes and clinical features. SSOBAR: Severely short of breath at rest. CARBOSE: Comfortable at rest breathless on slight exertion. This figure is illustrating possible causes and presentation of two distinct phenotypes of acute heart failure patients. 
reasons. These patients generally have neither a raised venous pressure nor peripheral oedema. Their problem is fluid in the wrong place (the lungs). Other relatively specific symptoms of heart failure are orthopnoea, and paroxysmal nocturnal dyspnoea. There are many other nonspecific features that may be due to heart failure, such as fatigue, disturbed sleep pattern, skeletal muscle wasting and depression, but these are generally unhelpful for its diagnosis.

\subsection{Physical Examination}

Examination of the lungs may reveal fine crepitations indicating pulmonary oedema. Crepitations are not an accurate guide to left ventricular filling pressure in patients with chronic heart failure, but may be clinically useful in the setting of acute heart failure for instance after a myocardial infarction. The Killip classification is a powerful prognostic tool in this clinical setting [14]. Most patients with chronic heart failure do not have lung crepitations even if left atrial pressure is increased, perhaps because of reduced permeability of the alveolar-capillary membrane or to increased pulmonary lymphatic clearance of fluid [1-17]. However, many patients have coarse crepitations due to pulmonary disease, some have fine crepitations due to pulmonary fibrosis and most patients who have rested in bed for a few hours will have some fine crepitations due to alveolar collapse that will resolve with a few coughs. Pulmonary crepitations in a patient who is not breathless at rest or on minimal exertion are unlikely to be due to heart failure.

A third heart sound may be normal in young people but indicates left ventricular dysfunction in people aged $>40$ years. The pulmonary component of the second heart sound (P2) will be increased in pulmonary hypertension, which may be secondary to left atrial hypertension. However, detection of these signs by auscultation has poor inter-observer reproducibility and is usually only obvious in patients with severe decompensation who are in sinus rhythm. Jugular venous pressure is one of the most specific signs of heart failure but often difficult to elicit, especially in a patient who is acutely breathless and using their accessory muscles of respiration. It reflects right atrial pressure and therefore will only be increased if there is a problem on the right side of the heart, which is a late manifestation of left sided heart disease [18]. Peripheral oedema is often present in patients with AHF but is usually a sign of late-stage disease and may be due to many other causes [13].

\subsection{Traditional Investigations}

The initial investigations performed in the acute setting are useful to assist in diagnosis, to identify precipitating factors and for risk stratification and triage for escalation to a high-dependency unit or transfer to a general ward or to an observation unit and same-day discharge (Figure 2). The ESC guidelines [1] strongly recommend an ECG, transthoracic echocardiography, measurement of blood chemistry and haematology and, less strongly, a chest X-ray and measurement of natriuretic peptides (BNP, NT-proBNP or MR-proANP). However, these recommendations are often not implemented in the acute setting even in expert units nor is it clear that they are

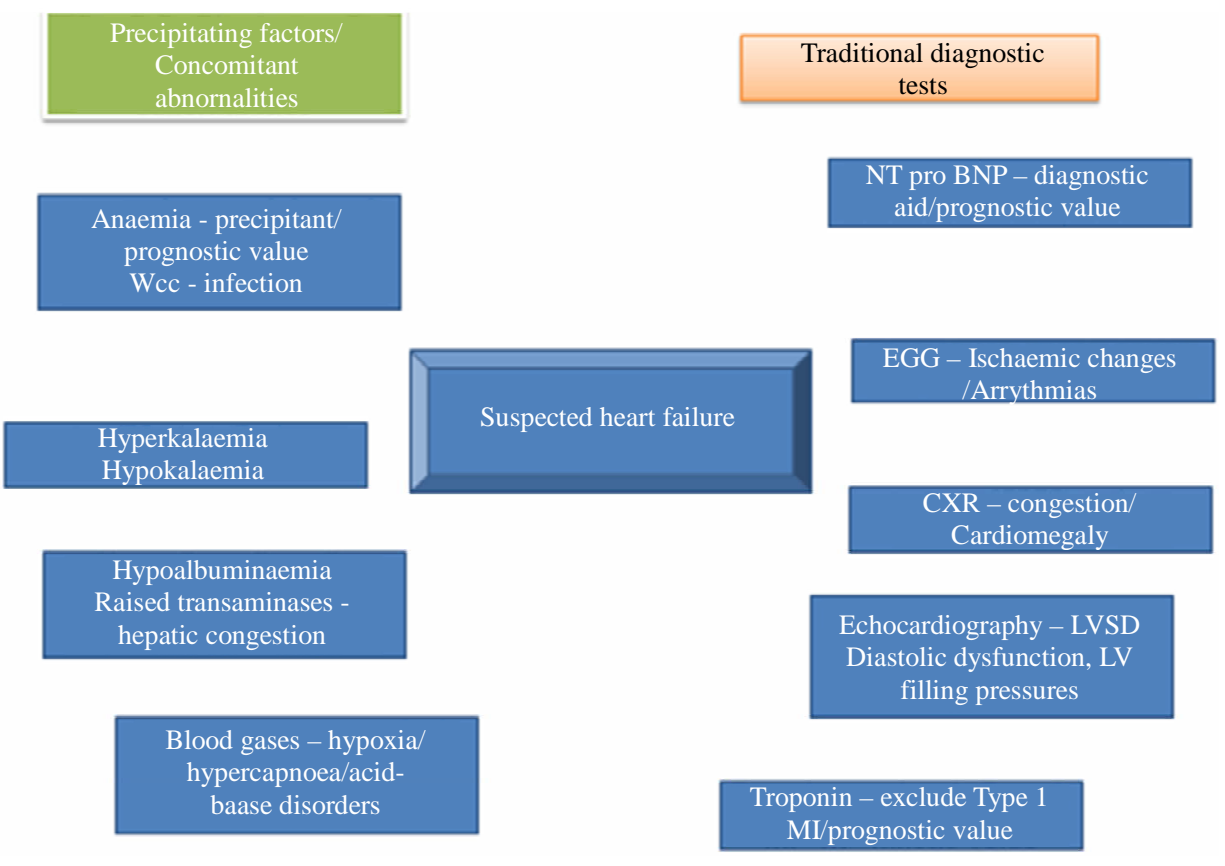

Figure 2. Standard diagnostic tests and possible abnormalities in acute heart failure. 
optimal. For instance, respiratory rate is probably the best method by which to quantify dyspnoea and yet it is often overlooked. Many authorities would consider it medical negligence not to order a chest X-ray for a patient with severe breathlessness to exclude pulmonary disease. Taking arterial gases is often painful for patients and entirely unnecessary. Trancutaneous oxygen saturations combined with venous gases provide all the necessary information. On the other hand, internationally, rather few acute medical receiving units have echocardiography available 24 hours a day, seven days per week.

\subsection{Natriuretic Peptides}

Natriuretic peptides can be considered cardiac stress hormones. Increases in natriuretic peptides are non-specific with respect to the nature of the stress but when normal are reassuring that the patient's cardiovascular system is not under great threat and when elevated that the patient has a problem requiring clarification. In the acute setting, measurement of natriuretic peptides may improve diagnostic accuracy by ruling out heart failure (NT-proBNP $<300 \mathrm{ng} / \mathrm{L}$ or BNP $<100 \mathrm{ng} / \mathrm{L}$ ) or by increasing the certainty of a clinical diagnosis (NT-proBNP > $2000 \mathrm{ng} / \mathrm{L}$ or BNP >500 ng/L). However, accurate interpretation requires experience and many patients will have a value in the diagnostic grey-zone $[19,20]$. The two main reasons for a substantial elevation in natriuretic peptides other than ventricular dysfunction are atrial fibrillation and renal dysfunction with sex (women have higher levels) and body mass index (fat people have lower levels) having a modest effect. Older people have higher levels but this reflects the decline in renal function and diastolic left ventricular function that occur with age. Older people have higher natriuretic peptides and a worse prognosis because their cardiac and renal function is worse. It is inappropriate to correct natriuretic peptides for age [21]. Natriuretic peptides do not discriminate between left ventricular systolic and diastolic dysfunction after correction for their impact on left atrial pressure, nor between right (e.g.: primary or secondary pulmonary hypertension) and left sided heart disease and cannot determine whether failure is due to intrinsic myocardial disease or due to excessive load on a fairly normal myocardium (e.g.: valve disease, malignant hypertension). In the IMPROVE CHF study, median plasma concentrations of NT-proBNP in patients with a final diagnosis of $\mathrm{HF}$ was $3697 \mathrm{ng} / \mathrm{L}$ as compared to $212 \mathrm{ng} / \mathrm{L}$ in those without HF [22]. In a very large registry of 48,629 patients, hospital mortality was more than three times higher in patients with BNP levels > $1730 \mathrm{ng} / \mathrm{L}$ (fourth quartile) as compared to those with levels $<430 \mathrm{ng} / \mathrm{L}$ (first quartile) [23].

Whether natriuretic peptides can be used to guide therapy in the setting of acute or chronic heart failure remains controversial [24,25].

\subsection{Adrenomedullin, Copeptin \& Procalcitonin}

In the BACH study, MR-proANP (>120 pmol/ml) was not inferior to BNP (>100 pg/ml) for identifying patients with heart failure and MR-pro ADM was superior to BNP and NT-proBNP for predicting 90-day mortality [26]. Copeptin and MR-proADM is probably a relatively non-specific marker of metabolic stress [27]. Their value may be in identifying patients who are likely to deteriorate and who require careful observation. Procalcitonin is a marker of infection, although also increased in heart failure, where it indicates an adverse prognosis. If it is elevated disproportionately to plasma concentrations of natriuretic peptides, it suggests that infection is an important component of the illness [28]. Thus, a panel of biomarkers combined with standard haematology and biochemistry profiles can build a patient profile that identifies diagnosis, precipitating factors and risk.

\subsection{Troponin}

Most patients with heart failure will have coronary artery disease as a cause or co-morbidity of heart failure and acute coronary syndromes (ACS) may often be a precipitating factor for exacerbations [10]. However, even patients with chronic stable heart failure commonly have elevated troponin levels and the great majority have measurable levels with the latest generation of assays. Diagnosis of ACS requires demonstration of a typical rise of troponin above the $99^{\text {th }}$ centile and, preferably, a subsequent fall. Patients with heart failure may be subject to what is termed Type II myocardial infarction (supply-demand mismatch rather than acute coronary obstruction), reflecting reduced subendocardial perfusion due to high left ventricular filling pressure, hypoxaemia, increases in afterload and or hypotension. This may lead to myocardial damage and accelerated cardiac myocyte apoptosis [29]. Troponin appears to be higher in patients with AHF (57\%) compared to patients with other causes of dyspnoea [29]. In the ADHERE registry, 75\% of patients with AHF had detectable troponin levels using an older generation assay although only $6.2 \%$ had values above the upper level of reference limit. This latter group of patients had, on average, lower ejection fractions and systolic blood pressure and a higher hospital mortality (8.0\% vs $2.7 \%$ ) [30]. Since the introduction of high sensitivity assays, it is possible to detect troponin in a large proportion of the healthy population [31]. Two recent clinical studies show that almost all patients with AHF had increases in high sensitivity troponin I or $\mathrm{T}$ and that increased levels predict poor in-patient and post-discharge prognosis [32,33]. In the PROTECT study, 21\% 
of patients were negative for Troponin at baseline but converted to detectable levels by Day 7 and these patients had higher mortality by 60 days [34]. Recently, the RELAX-AHF study showed that administration of the pregnancy-related vasodilator hormone serelaxin could reduce both plasma concentrations of natriuretic peptides and troponins and this effect appeared to be associated with an improvement in outcomes [35].

\subsection{Echocardiography}

Clinical guidelines recommend early echocardiography in acutely dyspnoeic patients who are suspected of having heart failure. This is a mostly unrealised ideal situation that rarely happens in clinical practice. Indeed, international research protocols in AHF have learnt to avoid requiring an immediate echocardiogram because that would preclude getting substantial numbers of patients into studies. There are too few adequately trained staff and little access to equipment to provide 24 hour cover seven days per week in most hospitals where these patients are seen (i.e. not University teaching hospitals!).

Ideally, prompt echocardiography should be part of the diagnostic work-up of all patients with suspected heart failure in the emergency setting, especially if a structural cause is suspected that might be amenable to intervention (e.g.: aortic stenosis, ruptured mitral chordae). Echocardiography will identify patients with heart failure and a reduced ejection fraction (HF-REF), although the severity of ventricular dysfunction is prone to substantial observer error. Ultrasound may also be used to assess lung oedema $[36,37]$. However, echocardiography is a highly inaccurate method of diagnosing heart failure with a preserved left ventricular ejection (HF-PEF) with the single most helpful echocardiographic measure being the left atrial volume or size. Natriuretic peptides are commonly grossly elevated in patients with HF-PEF and a fairly unremarkable looking echocardiogram. These patients respond symptomatically to treatment for heart failure and have a poor prognosis; so who are they if not patients with heart failure? Beware the false-negative echocardiogram in heart failure! Doppler echocardiography is complex to interpret, subject to many measurement errors and has failed the multi-centre clinical study test on many occasions. Regional wall motion abnormalities or thinning may indicate myocardial ischaemia or infarction but is usually unable to distinguish between acute and chronic ventricular dysfunction. Echocardiography is very useful for diagnosing valve problems and assessing RV function (tricuspid annular plane systolic excursion or TAPSE), pulmonary artery systolic pressure, inferior vena cava dilatation (indicating increased right atrial pressure), pericardial effusion and, with less confidence, constrictive pericarditis.

\section{NOVEL TECHNOLOGIES}

\subsection{Bio-Impedance}

From Ohms law, "when an electrical current is passed through human tissue, the voltage difference between two points on the body is proportional to impedance" [38]. Blood and solid organs that are fluid-rich offer lower impedance compared to bone and aerated lung and these principles can be used to assess hydration and haemodynamics by measuring fluctuations in signals that are proportional to the stroke volume and indices of myocardial contractility and relaxation [38].

Bio-impedance can be measured using external electrodes placed on wrists and ankles rather like a standard ECG or by electrode configurations that seek to measure thoracic impedance only (Figure 3). A major problem with non-invasive bio-impedance is the high impedance offered by skin. More recently, bio-impedance has been incorporated into implantable pacemakers and defibrillators which can then measure the impedance across the lung, bypassing the problem of skin impedance.

Using an implanted system, the MIDHeFT study reported that impedance monitoring could detect volume overload and predict the risk of hospitalisation with some success in a small single centre experience [39]. The larger, multi-centre FAST study, showed that impedance

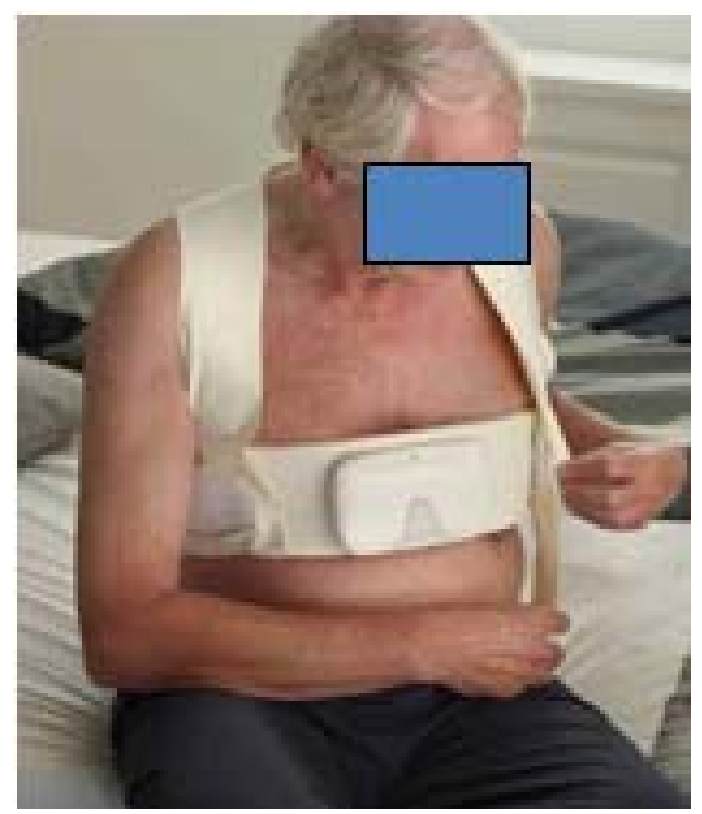

Figure 3. Bio-impedance monitoring is helpful in body fluid status evaluation in CHF patients. A: BioImpedonce Monitor (BIM) vest for patients with acutely decompensoted heart failure. Poster: Heart failure Congress 2012. Belgrade: Maayo 2012. Image proprierty of Philips Research. 
monitoring was more sensitive than weight gain in predicting future heart failure events (76\% vs 23\%) [40]. However, the DOT-HF study showed that providing patients with alerts increased the risk of hospitalisation [41]. Too many false positive alerts were observed for this technology to be considered viable [42].

There are too few data on the use of non-invasive bioimpedance to evaluate its diagnostic utility in the emergency setting or its usefulness for subsequent monitoring during the recovery phase. [38]. However, it is a simple, low-cost technology that offers many advantages. It can be used to monitor heart rate and rhythm and, fairly uniquely, respiratory rate. Studies have shown that the technology can track a reduction in lung fluid during diuresis $[43,44]$, cardiac output [45], predict decompensation in outpatients [46] and that it may carry independent prognostic information [47]. However, there is a great range of different bioimpedance equipment and in some studies it has been performed poorly [48]. More experience is required to understand its utility and accuracy [49].

\subsection{Remote Dielectric Sensing (ReDS)}

This technology uses either a wearable patch or implantable device that emits low-power electromagnetic signals into the chest $[50,51]$. Tissues reflect the signal according to their fluid content and this can be used to measure lung water. This might be used to predict deterioration or monitor resolution of lung oedema to identify the patient's optimal “dry” weight and timing of discharge.

\subsection{Pulse Wave Analysis (PWA)}

Pulse wave reflections can affect LV afterload and coronary perfusion [52] and may be important in the genesis of heart failure and its exacerbations. PWA, using noninvasive techniques such as applanation tonometry, may be used to assess systemic arterial stiffness and endothelial dysfunction (Figure 4). In the general population, abnormal arterial wave reflections predict incident cardiovascular events including the development of heart failure [52]. Sung and others, recently conducted pulse wave analysis shortly after admission for AHF and observed that abnormal wave reflections on admission predicted adverse events over the following 18 months, even after adjusting for other risk factors including NTproBNP [53]. Whether PWA can be used as a therapeutic target for existing and novel therapeutic interventions should be explored [54].

\subsection{Acoustic Cardiography (AC)}

Although the third heart sound (S3) may be specific for increased left ventricle filling pressure in adults and can

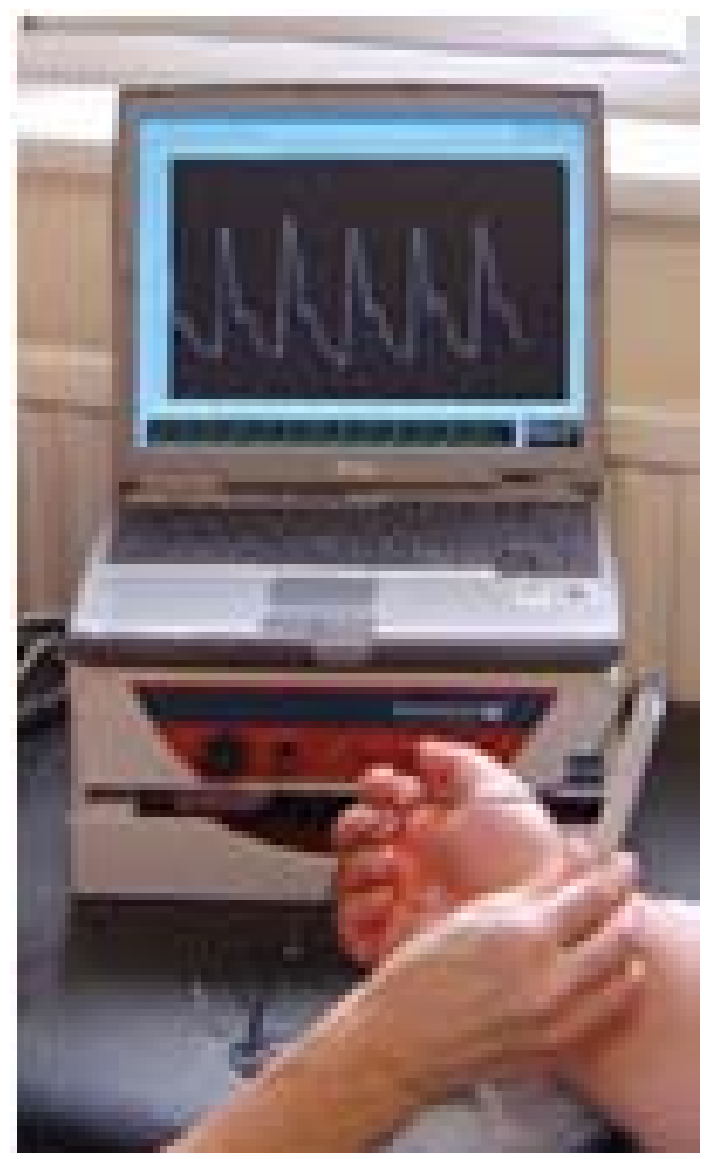

Figure 4. Pulse wave analysis. Pulse wave analysis: Image taken from November 2008 Volume 15, Issue 6 Br J Cardiol 2008; 15; 316-2.

predict outcome, it is often clinically difficult to detect in acute settings due to ambient noise, body habitues and tachypnoea. Expertise in auscultation will also vary greatly. Modern technological innovations now make it possible to capture information about S3 at the same time as the ECG recording (Figure 5) [55]. In the HEARD-IT trial, acoustic cardiography appeared helpful in risk stratification and diagnosis for patients with "grey zone" plasma concentrations of BNP. Patients with a "grey zone” BNP and an S3 by AC, were more likely to have a diagnosis of AHF confirmed subsequently and were more than twice as likely to have adverse events. AC is more sensitive in detecting an S3 than clinician auscultation whatever the body habitus [56] but may not provide clinically useful prognostic information independent of other readily available clinical variables [55].

\subsection{Finger Photoplethysmography (FPP)}

FPP can be used to measure the blood pressure (and heart rate) continuously, beat-to-beat (Figure 6). This feature alone may be useful for assessing patients with acute heart failure who are candidates for powerful old 

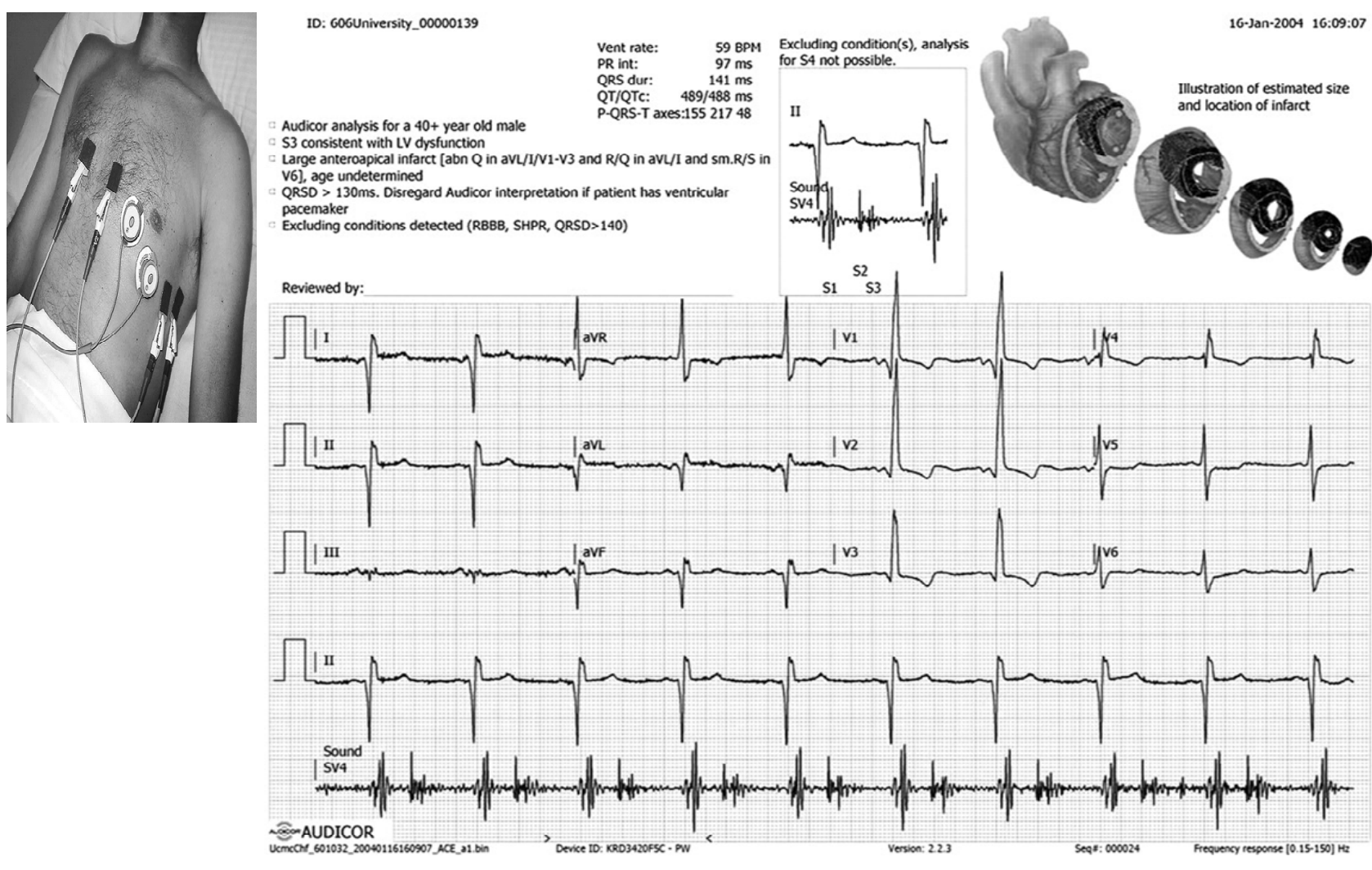

Figure 5. Acoustic cardiogrphy can capture third heart sound at same time during ECG recording.

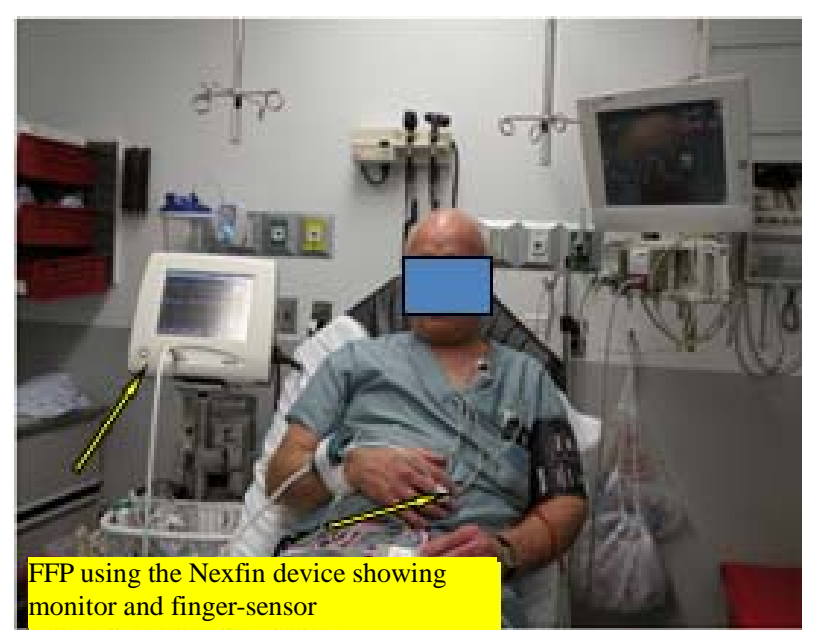

Figure 6. Finger photoplethsmography can measure beat to beat blood pressure, pulse, stroke volume, cardiac output and left ventricular end diastolic pressure. Image taken from the American Journal of Emergency Medicine volume 29, issue 7, September 2011, pages 782-789.

and new vasodilator agents that may cause profound hypotension [35]. Hypotensive episodes may not only cause distressing symptoms for the patient and an emergency situation for clinical staff but may cause renal dysfunction and further myocardial damage that have an adverse impact on longer term morbidity and mortality. However, FPP can also provide information on pulse volume which is a measure of stroke volume [57]. If heart rate, blood pressure and stroke volume are known, cardiac output and vascular resistance can be calculated, allowing haemodynamic therapies to be tailored to the individual patient's situation. Clinical assessment of LVEDP is not accurate in patients with AHF $[58,59]$. A good correlation between Pulse Amplitude Ratio (PAR) measured by FPP during a Valsalva manoeuvre and invasively measured Left Ventricle End Diastolic Pressure (LVEDP) has been reported [57]. Whether therapy guided to reduce LVEDP in patients with acute HF can reduce recurrent hospitalization is controversial $[48,59]$. Further studies with new technologies are needed.

\subsection{Swan-Ganz Catheter and Implantable Pressure Monitoring Devices}

This was the classic instrument for assessing and monitoring heart failure in the latter part of the $20^{\text {th }}$ Century. Many people doubted its utility. A large randomized trial demonstrated no advantage to direct measurement of pressures with treatment tailored to haemodynamic targets [60]. This may reflect the fact that haemodynamic decompensation depends not only on the absolute atrial 
pressure but also the rate of rise. Thus, a patient with chronic severe ventricular dysfunction may be relatively asymptomatic with a pulmonary capillary wedge pressure of $20 \mathrm{mmHg}$ and a patient with new onset cardiac dysfunction may be in pulmonary oedema with a wedge pressure of $15 \mathrm{mmHg}$. Invasive haemodynamic monitoring might be of value in complex cases (for instance patients who have significant lung disease or those with severe right ventricular dysfunction) or when arterial pressure is low to ensure that the ventricular filling pressure is not reduced excessively resulting in a fall in cardiac output and the development of hypotension and shock. More recently, chronically implantable pulmonary artery pressure monitors have been developed [9]. Preliminary evidence suggests that increases in pulmonary artery pressure predict the risk of decompensation and that treating pulmonary artery pressures reduces the risk of decompensation. Pressure monitors that screw into the atrial septum that can monitor left atrial pressure are also being developed [61].

\subsection{Coronary Angiography}

In acute HF, current US guidelines recommend urgent cardiac catheterization and attempt to revascularization when prolonged meaningful survival is expected in patients with known or suspected myocardial ischaemia, especially when there are clinical features of hypoperfusion [62]. Neither the safety nor efficacy of this recommendation has been established nor is it currently practically feasible in most clinical settings where these patients are managed. European guidelines are less dogmatic on this issue! This may reflect publication of two substantial randomised trials of revascularisation in patients with chronic heart failure, neither of which showed a benefit on mortality $[63,64]$.

\section{CONCLUSION}

The management of AHFS is often poor and this may explain, in part, its dire prognosis. Despite of limitations, clinical evaluation remains the primary tool for initial assessment and monitoring the response to therapy. A single measurement of plasma concentrations of a natriuretic peptide provides reassurance that a clinical diagnosis of AHFS is correct; normal concentrations alert the clinician to rare diagnoses, such as constrictive pericarditis, or alternative diagnoses mimicking heart failure. However, natriuretic peptides are not diagnostic when used alone, nor has it been established that serial measurement of natriuretic peptides adds value to clinical monitoring. Further studies are required to assess the practical clinical value of applying novel technologies to the management of AHF to monitor the response to treatment, discharge readiness and the risk of readmission.
However, monitoring will not help patients unless it changes management. Ultimately, novel technologies need to show that they change decisions about care made by clinicians and/or patients, leading to more favourable outcomes including better symptom control, less disability and a longer life. If, as a by-product, they can also reduce the frequency or shorten the duration of hospitalisation, a surrogate measure of patient well-being and health-service costs, then so much the better.

\section{REFERENCES}

[1] McMurray, J.J., et al. (2012) ESC Guidelines for the diagnosis and treatment of acute and chronic heart failure 2012: The Task Force for the Diagnosis and Treatment of Acute and Chronic Heart Failure 2012 of the European Society of Cardiology. Developed in collaboration with the Heart Failure Association (HFA) of the ESC. European Heart Journal, 33, 1787-1847. doi:10.1093/eurheartj/ehs104

[2] Johansson, S., et al. (2001) Incidence of newly diagnosed heart failure in UK general practice. European Journal of Heart Failure, 3, 225-231. doi:10.1016/S1388-9842(00)00131-8

[3] Cleland, J.G., et al. (2011) The national heart failure audit for England and Wales 2008-2009. Heart, 97, 876-886. doi:10.1136/hrt.2010.209171

[4] Dharmarajan, K., et al. (2013) Diagnoses and timing of 30-day readmissions after hospitalization for heart failure, acute myocardial infarction, or pneumonia. JAMA, 309, 355-363. doi:10.1001/jama.2012.216476

[5] Nieminen, M.S., et al. (2006) EuroHeart Failure Survey II (EHFS II): A survey on hospitalized acute heart failure patients: Description of population. European Heart Journal, 27, 2725-2736. doi:10.1093/eurheartj/ehl193

[6] Howlett, J.G. (2011) Acute heart failure: Lessons learned so far. Canadian Journal of Cardiology, 27, 284-295. doi:10.1016/j.cjca.2011.02.007

[7] Shoaiba, D.B., Mabote, T., Pellicori, P., Torabi, A., Clark, A., Wong, K. and Cleland, J.G. (2013) Checking-up on the National Audit on Heart Failure: Are only a minority of patients admitted with heart failure truly breathless at rest and how many are suitable for Serelaxin? BCS Annual Conference, London, 3-5 June 2013.

[8] Inglis, S.C., et al. (2011) Which components of heart failure programmes are effective? A systematic review and meta-analysis of the outcomes of structured telephone support or telemonitoring as the primary component of chronic heart failure management in 8323 patients: Abridged cochrane review. European Journal of Heart Failure, 13, 1028-1040. doi:10.1093/eurjhf/hfr039

[9] Abraham, W.T., et al. (2011) Wireless pulmonary artery haemodynamic monitoring in chronic heart failure: A randomised controlled trial. Lancet, 377, 658-666. doi:10.1016/S0140-6736(11)60101-3

[10] Adamson, P.B. (2005) Ambulatory hemodynamics in patients with chronic heart failure: implications for vol- 
ume management in elderly patients. The American Journal of Geriatric Cardiology, 14, 236-241. doi:10.1111/j.1076-7460.2005.02596.x

[11] Cleland, J.G., et al. (2009) Clinical trials update from the European Society of Cardiology Meeting 2009: AAA, RELY, PROTECT, ACTIVE-I, European CRT survey, German pre-SCD II registry, and MADIT-CRT. European Journal of Heart Failure, 11, 1214-1219. doi:10.1093/eurjhf/hfp162

[12] Cleland, J., et al. (2009) Outcome of patients discharged on loop diuretic therapy with or without diagnosis of Heart Failure. European Heart Failure, Nice, 30 May-2 June 2009.

[13] Nohria, A., Mielniczuk, L.M. and Stevenson, L.W. (2005) Evaluation and monitoring of patients with acute heart failure syndromes. American Journal of Cardiology, 96, 32G-40G. doi:10.1016/j.amjcard.2005.07.019

[14] Parakh, K., et al. (2008) Long-term significance of Killip class and left ventricular systolic dysfunction. American Journal of Medicine, 121, 1015-1018. doi:10.1016/j.amjmed.2008.06.020

[15] Clark, A.L. and Cleland, J.G. (2013) Causes and treatment of oedema in patients with heart failure. Nature Reviews Cardiology, 10, 156-170. doi:10.1038/nrcardio.2012.191

[16] Smith, A.A., et al. (1999) Impaired pulmonary diffusion during exercise in patients with chronic heart failure. Circulation, 100, 1406-1410. doi:10.1161/01.CIR.100.13.1406

[17] Puri, S., et al. (1995) Reduced alveolar-capillary membrane diffusing capacity in chronic heart failure. Its pathophysiological relevance and relationship to exercise performance. Circulation, 91, 2769-2674. doi:10.1161/01.CIR.91.11.2769

[18] Drazner, M.H., et al. (2012) Relationship of right- and left-sided filling pressures in patients with advanced heart failure: A 14-year multi-institutional analysis. The Journal of Heart and Lung Transplantation, 31, 67-72. doi:10.1016/j.healun.2011.09.003

[19] Maisel, A., et al. (2008) State of the art: using natriuretic peptide levels in clinical practice. European Journal of Heart Failure, 10, 824-839. doi:10.1016/j.ejheart.2008.07.014

[20] Januzzi, J.L., et al. (2006) NT-proBNP testing for diagnosis and short-term prognosis in acute destabilized heart failure: An international pooled analysis of 1256 patients: The International Collaborative of NT-proBNP Study. European Heart Journal, 27, 330-337. doi:10.1093/eurheartj/ehi631

[21] Frankenstein, L., et al. (2009) The prognostic value of individual NT-proBNP values in chronic heart failure does not change with advancing age. Heart, 95, 825-829. doi:10.1136/hrt.2008.158626

[22] Moe, G.W., et al. (2007) N-terminal pro-B-type natriuretic peptide testing improves the management of patients with suspected acute heart failure: Primary results of the Canadian prospective randomized multicenter IMPROVE-CHF study. Circulation, 115, 3103-3110.

\section{doi:10.1161/CIRCULATIONAHA.106.666255}

[23] Fonarow, G.C., et al. (2007) Admission B-type natriuretic peptide levels and in-hospital mortality in acute decompensated heart failure. Journal of the American College of Cardiology, 49, 1943-1950. doi:10.1016/j.jacc.2007.02.037

[24] Felker, G.M., et al. (2009) Biomarker-guided therapy in chronic heart failure: A meta-analysis of randomized controlled trials. American Heart Journal, 158, 422-430. doi:10.1016/j.ahj.2009.06.018

[25] Singer, A.J., et al. (2009) Rapid Emergency Department Heart Failure Outpatients Trial (REDHOT II): A randomized controlled trial of the effect of serial B-type natriuretic peptide testing on patient management. Circulation: Heart Failure, 2, 287-293. doi:10.1161/CIRCHEARTFAILURE.108.826685

[26] Maisel, A., et al. (2010) Mid-region pro-hormone markers for diagnosis and prognosis in acute dyspnea: Results from the BACH (Biomarkers in Acute Heart Failure) trial. Journal of the American College of Cardiology, 55, 2062 2076. doi:10.1016/j.jacc.2010.02.025

[27] Peacock, W.F., et al. (2011) Short-term mortality risk in emergency department acute heart failure. Academic Emergency Medicine, 18, 947-958. doi:10.1111/j.1553-2712.2011.01150.x

[28] Maisel, A., et al. (2012) Use of procalcitonin for the diagnosis of pneumonia in patients presenting with a chief complaint of dyspnoea: Results from the BACH (Biomarkers in Acute Heart Failure) trial. European Journal of Heart Failure, 14, 278-286. doi:10.1093/eurjhf/hfr177

[29] Januzzi Jr., J.L., et al. (2012) Troponin elevation in patients with heart failure: On behalf of the third Universal Definition of Myocardial Infarction Global Task Force: Heart Failure Section. European Heart Journal, 33, 22652271. doi:10.1093/eurheartj/ehs191

[30] Peacock, W.F., et al. (2008) Cardiac troponin and outcome in acute heart failure. The New England Journal of Medicine, 358, 2117-2126. doi:10.1056/NEJMoa0706824

[31] Twerenbold, R., et al. (2012) High-sensitive troponin T measurements: What do we gain and what are the challenges? European Heart Journal, 33, 579-586. doi:10.1093/eurheartj/ehr492

[32] Xue, Y., et al. (2011) Serial changes in high-sensitive troponin I predict outcome in patients with decompensated heart failure. European Journal of Heart Failure, 13, 37-42. doi:10.1093/eurjhf/hfq210

[33] Pascual-Figal, D.A., et al. (2011) Soluble ST2, high-sensitivity troponin $\mathrm{T}$ - and N-terminal pro-B-type natriuretic peptide: Complementary role for risk stratification in acutely decompensated heart failure. European Journal of Heart Failure, 13, 718-725. doi:10.1093/eurjhf/hfr047

[34] O’Connor, C.M., et al. (2011) Impact of serial troponin release on outcomes in patients with acute heart failure: Analysis from the PROTECT pilot study. Circulation: Heart Failure, 4, 724-732. doi:10.1161/CIRCHEARTFAILURE.111.961581

[35] Metra, M., et al. (2013) Effect of serelaxin on cardiac, renal, and hepatic biomarkers in the Relaxin in Acute 
Heart Failure (RELAX-AHF) development program: Correlation with outcomes. Journal of the American College of Cardiology, 61, 196-206.

[36] Platz, E., et al. (2012) Utility of lung ultrasound in predicting pulmonary and cardiac pressures. European Journal of Heart Failure, 14, 1276-1284.

doi:10.1093/eurjhf/hfs144

[37] Picano, E. and Gargani, L. (2012) Ultrasound lung comets: The shape of lung water. European Journal of Heart Failure, 14, 1194-1196.

[38] Tang, W.H. and Tong, W. (2009) Measuring impedance in congestive heart failure: Current options and clinical applications. American Heart Journal, 157, 402-411. doi:10.1016/j.ahj.2008.10.016

[39] Yu, C.M., et al. (2005) Intrathoracic impedance monitoring in patients with heart failure: Correlation with fluid status and feasibility of early warning preceding hospitalization. Circulation, 112, 841-848. doi:10.1161/CIRCULATIONAHA.104.492207

[40] Abraham, W.T., et al. (2011) Intrathoracic impedance vs daily weight monitoring for predicting worsening heart failure events: Results of the Fluid Accumulation Status Trial (FAST). Congestive Heart Failure, 17, 51-55. doi:10.1111/j.1751-7133.2011.00220.x

[41] van Veldhuisen, D.J., et al.(2011) Intrathoracic impedance monitoring, audible patient alerts, and outcome in patients with heart failure. Circulation, 124, 1719-1726. doi:10.1161/CIRCULATIONAHA.111.043042

[42] Cleland, J.G. and Antony, R. (2011) It makes SENSE to take a safer road. European Heart Journal, 32, 22252227. doi:10.1093/eurheartj/ehr120

[43] Freimark, D., et al. (2007) Monitoring lung fluid content in CHF patients under intravenous diuretics treatment using bio-impedance measurements. Physiological Measurement, 28, S269-S277. doi:10.1088/0967-3334/28/7/S20

[44] Arad, M., et al. (2009) Estimating pulmonary congestion in elderly patients using bio-impedance technique: Correlation with clinical examination and X-ray results. Medical Engineering \& Physics, 31, 959-963. doi:10.1016/j.medengphy.2009.05.008

[45] Northridge, D.B., et al. (1990) Non-invasive determination of cardiac output by Doppler echocardiography and electrical bioimpedance. British Heart Journal, 63, 93-97. doi:10.1136/hrt.63.2.93

[46] Packer, M., et al. (2006) Utility of impedance cardiography for the identification of short-term risk of clinical decompensation in stable patients with chronic heart failure. Journal of the American College of Cardiology, 47, 2245-2252. doi:10.1016/j.jacc.2005.12.071

[47] Malfatto, G., et al. (2013) Transthoracic bioimpedance and brain natriuretic Peptide assessment for prognostic stratification of outpatients with chronic systolic heart failure. Clinical Cardiology, 36, 103-109. doi:10.1002/clc. 22086

[48] Kamath, S.A., et al. (2009) Correlation of impedance cardiography with invasive hemodynamic measurements in patients with advanced heart failure: the BioImpedance
CardioGraphy (BIG) substudy of the evaluation study of congestive heart failure and pulmonary artery catheterization effectiveness (ESCAPE) trial. American Heart Journal, 158, 217-223. doi:10.1016/j.ahj.2009.06.002

[49] Di Somma, S., et al. (2012) The emerging role of biomarkers and bio-impedance in evaluating hydration status in patients with acute heart failure. Clinical Chemistry and Laboratory Medicine, 50, 2093-2105.

[50] Amir, O., et al. (2013) A novel approach to monitoring pulmonary congestion in heart failure: Initial animal and clinical experiences using remote dielectric sensing technology. Congestive Heart Failure, in press.

[51] Gal T Ben, A.O., Rappaport, D., Rekant, K., Adamson, P.B., Anker, S., Cleland, J.G., Jaarsma, T. and Abraham, W.T. (2013) A pilot study on the feasibility of heart failure medical management based on daily monitoring of pulmonary subclinical congestion with ReDS technology in outpatient set up. ESC Heart Failure, Lisbon, 25-28 May 2013.

[52] Chirinos, J.A., et al. (2012) Arterial wave reflections and incident cardiovascular events and heart failure: MESA (Multiethnic Study of Atherosclerosis). Journal of the American College of Cardiology, 60, 2170-2177. doi:10.1016/j.jacc.2012.07.054

[53] Sung, S.H., et al. (2012) Excessive wave reflections on admission predict post-discharge events in patients hospitalized due to acute heart failure. European Journal of Heart Failure, 14, 1348-1355. doi:10.1093/eurjhf/hfs124

[54] Shah, S.J. and Wasserstrom, J.A. (2012) Increased arterial wave reflection magnitude: A novel form of stage B heart failure? Journal of the American College of Cardiology, 60, 2178-2181. doi:10.1016/j.jacc.2012.07.055

[55] Collins, S.P., et al. (2009) S3 detection as a diagnostic and prognostic aid in emergency department patients with acute dyspnea. Annals of Emergency Medicine, 53, 748757. doi:10.1016/j.annemergmed.2008.12.029

[56] Maisel, A.S., et al. (2011) Acoustic cardiography S3 detection use in problematic subgroups and B-type natriuretic peptide "gray zone": Secondary results from the heart failure and audicor technology for rapid diagnosis and initial treatment multinational investigation. American Journal of Emergency Medicine, 29, 924-931. doi:10.1016/j.ajem.2010.03.032

[57] Silber, H.A., et al. (2012) Finger photoplethysmography during the Valsalva maneuver reflects left ventricular filling pressure. American Journal of Physiology Heart and Circulatory Physiology, 302, H2043-H2047. doi:10.1152/ajpheart.00609.2011

[58] Gheorghiade, M., et al. (2007) Short-term clinical effects of tolvaptan, an oral vasopressin antagonist, in patients hospitalized for heart failure: The EVEREST clinical status trials. The Journal of the American Medical Association, 297, 1332-1343. doi:10.1001/jama.297.12.1332

[59] Sharma, G.V., et al. (2011) Noninvasive monitoring of left ventricular end-diastolic pressure reduces rehospitalization rates in patients hospitalized for heart failure: A randomized controlled trial. Journal of Cardiac Failure, 17, 718-725. doi:10.1016/j.cardfail.2011.04.014 
[60] Yamokoski, L.M., et al. (2007) Prediction of rehospitalization and death in severe heart failure by physicians and nurses of the ESCAPE trial. Journal of Cardiac Failure, 13, 8-13. doi:10.1016/j.cardfail.2006.10.002

[61] Ritzema, J.L., et al. (2011) Serial Doppler echocardiography and tissue Doppler imaging in the detection of elevated directly measured left atrial pressure in ambulant subjects with chronic heart failure. JACC: Cardiovascular Imaging, 4, 927-934. doi:10.1016/j.jcmg.2011.07.004

[62] Hunt, S.A., et al. (2009) 2009 Focused update incorporated into the ACC/AHA 2005 guidelines for the diagnosis and management of heart failure in adults: A report of the American college of cardiology foundation/American
Heart Association Task Force on practice guidelines developed in collaboration with the International society for heart and lung transplantation. Journal of the American College of Cardiology, 53, e1-e90. doi:10.1016/j.jacc.2008.11.013

[63] Doenst, T., et al. (2013) Influence of crossover on mortality in a randomized study of revascularization in patients with systolic heart failure and coronary artery disease. Circulation Heart Failure, 6, 445-450.

[64] Cleland, J.G., et al. (2011) The heart failure revascularisation trial (HEART). European Journal of Heart Failure, 13, 227-233. doi:10.1093/eurjhf/hfq230 